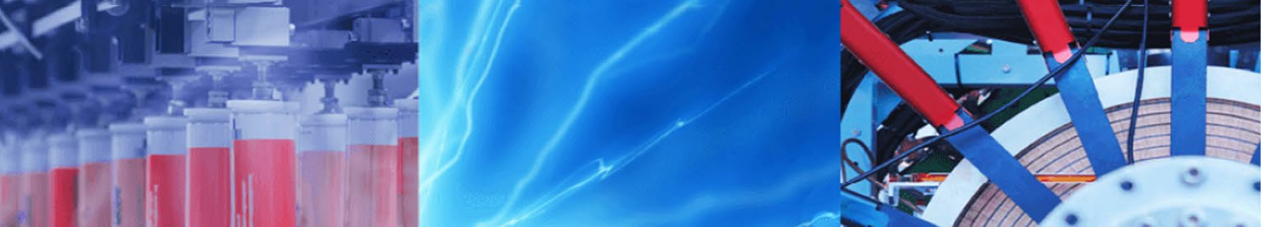

Short Communication

\title{
A simplified thermomechanical approach to visualize hexagonal honeycomb construction
}

\author{
Dhritiman Talukdar ${ }^{1} \cdot$ Kishore Dutta $^{1}$ (D)
}

(c) Springer Nature Switzerland AG 2019

\begin{abstract}
The construction of honeycombs astonished human being from ancient time due to their perfect geometrical structure. How the hexagonal cells are constructed from fresh circular cells during the cell building process is a matter of long debate. Here we show solely from the thermomechanical properties of self-synthesized beeswax how the honeybees permanently transform the fresh circular cells to rounded hexagons by creating a temperature gradient in the vicinity of the triple junctions of circular cell walls. By assuming conventional Fourier's law of heat conduction and mechanical properties of beeswax, we show via computer simulations that with increasing temperature gradient, the stress on the adjacent circular cell walls increases due to enhanced fusion, leading to the deformation from the original positions of the circular cell walls. The calculated von Mises stresses are found to be significantly larger than the yield stress, indicating the permanent deformation of the circular cell walls to rounded hexagonal shape. This suggests that it is the thermomechanical properties of the building material for which the comb cells take rounded hexagonal shapes.
\end{abstract}

Keywords Beeswax $\cdot$ Honeycomb $\cdot$ Rounded hexagon $\cdot$ Fourier's law $\cdot$ Von Misses stress

\section{Introduction}

One of the spectacular architectures with astounding precision that fascinated natural philosophers for centuries is the nest of honeybees [1-5]. How do honeybees build their comb cells in the shape of hexagons without the assistance of geometrical tools such as protectors or compasses? Although there has been a number of explanations in the literature, the extreme precision with which the honeybees construct the honeycomb has long been remained as a mystery. The honeycomb cells are constructed from self-synthesized wax secreted by worker bees. Cells are not hexagonal when they are built but rather circular, as already suspected by observation and easily demonstrated by studying wax comb in the early stage of construction [6-8]. One of the explanations for designing perfect hexagonal shape from fresh circular cells is based on liquid equilibrium hypothesis
[6]. As observations suggest that the thermal sources of increased wax temperature are the individual hot worker bees $[9,10]$, according to the viewpoint of Pirk et al. [6], bees' body heat increases the temperature in the vicinity of the cylindrical hole until the wax reaches a liquid equilibrium state, after which simple mechanical surface tension causes the circular frame to be hexagonal. In the numerical study [7], Karihaloo proved that, under the liquid equilibrium hypothesis that results from surface tension, the soften wax could be thinned so that the hexagonal frame would appear spontaneously. More precisely, the molten viscoelastic wax, being heated by hot worker bees, flows near the triple junction between neighboring circular cells that transform circular honeycomb cells in a natural honeybee comb quickly into rounded hexagonal structure. However, this liquid equilibrium hypothesis undergoes a serious objection [11] where it has been argued on the basis of experimental observations that bees do not heat

Kishore Dutta, kdkishore77@gmail.com; Dhritiman Talukdar, dhritimant@gmail.com | 'Department of Physics, Handique Girls' College, Guwahati 781 001, India.

SN Applied Sciences (2019) 1:1220 | https://doi.org/10.1007/s42452-019-1239-0 
up wax to the temperatures needed for the wax to reach the liquid equilibrium. If this is so, is there any other mechanism through which circular cells transform to hexagonal shapes without reaching the melting point?

Here we demonstrate by using only thermal and mechanical properties of beeswax in computer simulation that a measurable deformation of the circular cell walls takes place if a finite temperature gradient is maintained in the vicinity of the triple junctions. Due to the heat generated by the worker bees, the temperature gradient so produced is enough to generate stress on the adjacent circular walls that gradually increases the contact area of the curved walls and make them planar. The calculated von Mises stresses on the rims are found to be higher than the yield stress of beeswax, indicating permanent deformation of the circular cell walls to rounded hexagonal shape. This indicates that it is the inherent properties of beeswax for which under thermal expansion the circular cells transform into hexagonal cells in a honeycomb.

\section{Materials and methods}

In order to visualize the structural change, we follow the work of Ref. [7] and, as shown in Fig. 1, construct a triple junction from three isodiametric circular cells with radius $R_{0}=3 \mathrm{~mm}$ and thickness $h=90 \mu \mathrm{m}$, as measured in Ref. [12]. In such a computational geometry, consisting of total 24 surfaces as displayed in Fig. 2, we use a very fine mesh having 50,448 number of elements. The junction of two adjacent circular cell walls is filled up with small surfaces labeled as 16-24 in Fig. 2. It is these junctions which are gradually filled by the bees with hot wax flakes. The mechanical properties of beeswax have

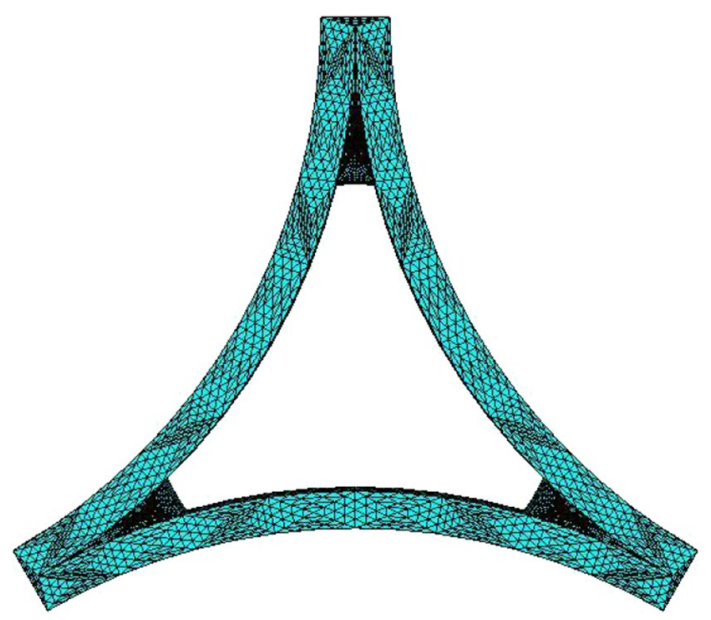

Fig. 1 Computational mesh for a triple junction constructed from three adjacent isodiametric circular cell walls of radius $3 \mathrm{~mm}$ and thickness $90 \mu \mathrm{m}$

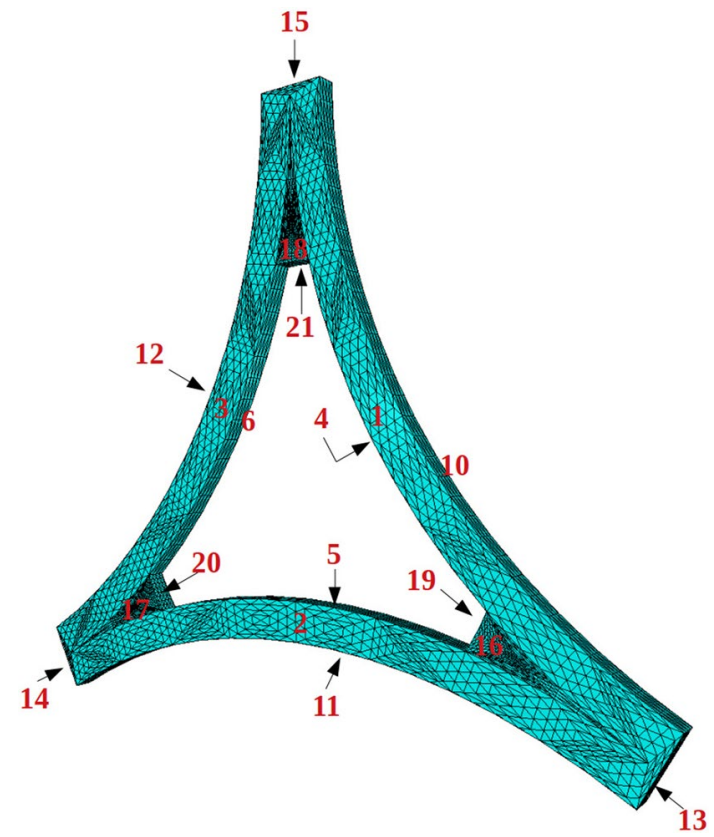

Fig. 2 Computational mesh of the triple junction shown in Fig. 1 consists of total 24 surfaces. Surfaces labeled as 7, 8, 9 are just on the opposite side of the surfaces 1, 2, 3. Similarly, surfaces 22-24 are on the opposite sides of 16-18

a great influence on the properties such as the strength and stiffness of the final structure. The parameters representing the mechanical and thermal properties of honeybees' wax vary from species to species [2, 9, 12-14]. For the present simulation, we take their values from various references $[12,14,15]$. These are the coefficient of thermal expansion $\beta=344 \times 10^{-6} \mathrm{~K}^{-1}$, thermal conductivity $\kappa=0.4 \mathrm{~W} \mathrm{~m}^{-1} \mathrm{~K}^{-1}$, heat capacity $c_{\mathrm{p}}=2025 \mathrm{~J} \mathrm{~kg}^{-1}$, density $\rho=970 \mathrm{~kg} \mathrm{~m}^{-3}$, Poisson's ratio $v=0.30$, and Young's modulus $Y=39 \times 10^{6} \mathrm{~Pa}$. Using these values, we prepare the beeswax as a material for the computational geometry.

After filling up the computational mesh with the beeswax, we create a temperature difference by imposing Dirichlet boundary conditions on the surfaces. In order to do this, we follow the available experimental observations $[6,9$, $10]$, suggesting that the temperature is relatively high (from 303 to $318 \mathrm{~K}$ ) in areas where the building activity takes place in comparison with the surrounding non-construction areas. As such, the three extreme corner surfaces labeled as 13-15 in Fig. 2 are held fixed at a constant room temperature of $295 \mathrm{~K}$. The three outer rims of the circular walls labeled as $10-12$ in Fig. 2 are also maintained at room temperature ( $295 \mathrm{~K}$ ), while the surfaces labeled as 16-24 in the same figure are maintained at a relatively high temperature (308 K). This temperature of the hot surfaces is chosen in such a way that it lies within the temperature range observed in experiment during the building activity of the beeswax [11]. Such a temperature difference initiates conduction of heat in the 
vicinity of the triple junction. For simplicity, we assume that the heat conduction takes place obeying Fourier law and incompressible heat equation, given by

$\rho c_{\mathrm{p}} \frac{\partial T}{\partial t}=\nabla \cdot(\kappa \nabla T)$,

where $\rho$ is the density, $c_{\mathrm{p}}$ is the heat capacity at constant pressure, $T$ is the temperature, and $\kappa$ is the thermal conductivity. The resulting distribution of temperature field over the entire triple junction is displayed in Fig. 3.

Since beeswax possesses both elastic and plastic behaviors [9], there will be finite deformation due to stress generated in the presence of temperature gradient. The stress tensor $\tau_{i j}$ is given by

$\tau_{i j}=C^{i j k l} \varepsilon_{k l}-\beta^{i j}\left(T-T_{0}\right)$,

where $\varepsilon$ is the strain, $\beta$ is the heat expansion coefficient, $T$ is the temperature field, $T_{0}$ is the reference temperature of the stress-free state, and $C$ is the stiffness matrix which involves the Young's modulus and the Poisson's ratio. The stress tensor $\tau_{i j}$ consists of nine components that completely define the state of stress at a point inside the material in the deformed state. The resulting stress, in turn, generates displacement governed by the dynamical equation for elastic deformation of solids, given by

$\rho \frac{\partial^{2} \mathbf{d}}{\partial^{2} t}=\nabla \cdot \tau$

where $\rho$ is the density and $\mathbf{d}$ is the displacement field. The linearized strains can be related to the displacement through the expression

$\varepsilon=\frac{1}{2}\left[\nabla \mathbf{d}+(\nabla \mathbf{d})^{T}\right]$.

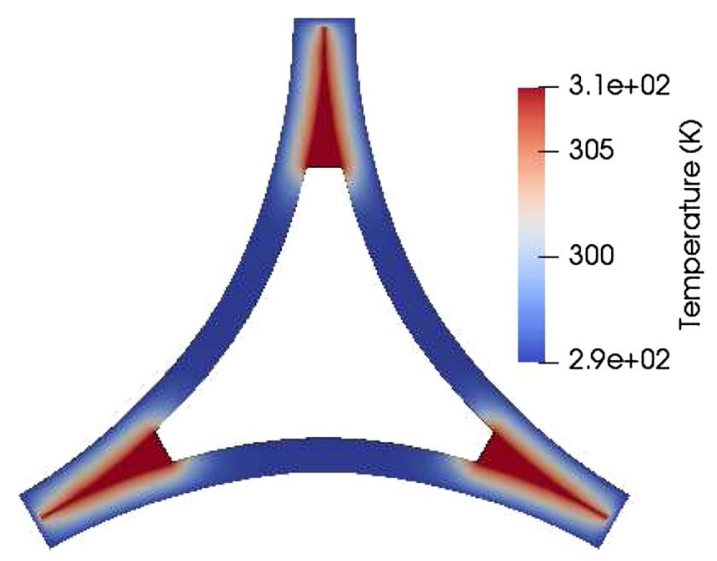

Fig. 3 Distribution of temperature field in the vicinity of the triple junction where the hot junctions are maintained at $308 \mathrm{~K}$ and the cold surfaces are at $295 \mathrm{~K}$. The color bar indicates the temperature scale in Kelvin
Further, in order to check the nature of deformation of the circular rims, it is essential to measure von Misses stress and then to compare with yield strength of beeswax. Yield strength is defined as the stress at which a material begins to deform plastically. The von Mises stress is just a metric of measurement to determine whether the structure has started to yield at any point [16]. According to von Mises criterion, a given structural material is safe as long as the maximum value of the distortion energy per unit volume in that material remains smaller than the distortion energy per unit volume required to cause yield. In other words, the deformed state will be permanent if the von Misses stress is higher than the yield stress of the material. The von Misses stress $\tau_{v}$ is calculated using the relation

$\tau_{v}=\sqrt{\frac{\left(\tau_{11}-\tau_{22}\right)^{2}+\left(\tau_{22}-\tau_{33}\right)^{2}+\left(\tau_{33}-\tau_{11}\right)^{2}+6\left(\tau_{12}^{2}+\tau_{23}^{2}+\tau_{31}^{2}\right)}{2}}$.

\section{Simulation results}

The finite temperature difference maintained in the vicinity of the triple junction creates stress on the fresh circular cell walls. This can be visualized from Fig. 4 where the circular rims undergo finite displacements when the hot junctions are maintained at $308 \mathrm{~K}$. The maximum displacement magnitude occurs at the middle of the rim for each run. To see how the stress increases with temperature, we run the simulation by maintaining the hot junctions at different temperatures $(318,328,338,348$, and $358 \mathrm{~K})$, while the temperature of the cold regions remains unchanged (room temperature, $295 \mathrm{~K}$ ). As displayed in the graphical plot of Fig. $6 \mathrm{a}$, the magnitudes of the resulting maximum

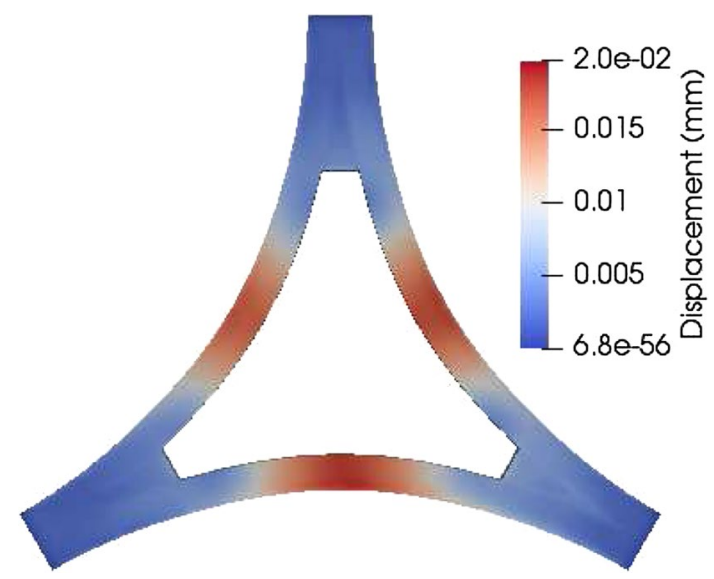

Fig. 4 Deformation of the circular cell walls due to temperature distribution shown in Fig. 3. The contraction of adjacent rims is visible as indicated by the color bar, showing the displacement magnitude in $\mathrm{mm}$ 
displacement are found to increase with the temperature of the hot junctions.

We then calculate von Mises stress both at the rim and at the meniscus. When the hot surfaces are maintained at $308 \mathrm{~K}$, we obtain the distribution of von Mises stress as displayed in Fig. 5. The values of von Mises stress at the meniscus and at the rims are found to be $4.9 \mathrm{MPa}$ and 5.27 MPa, respectively. As shown in the graphical plots of

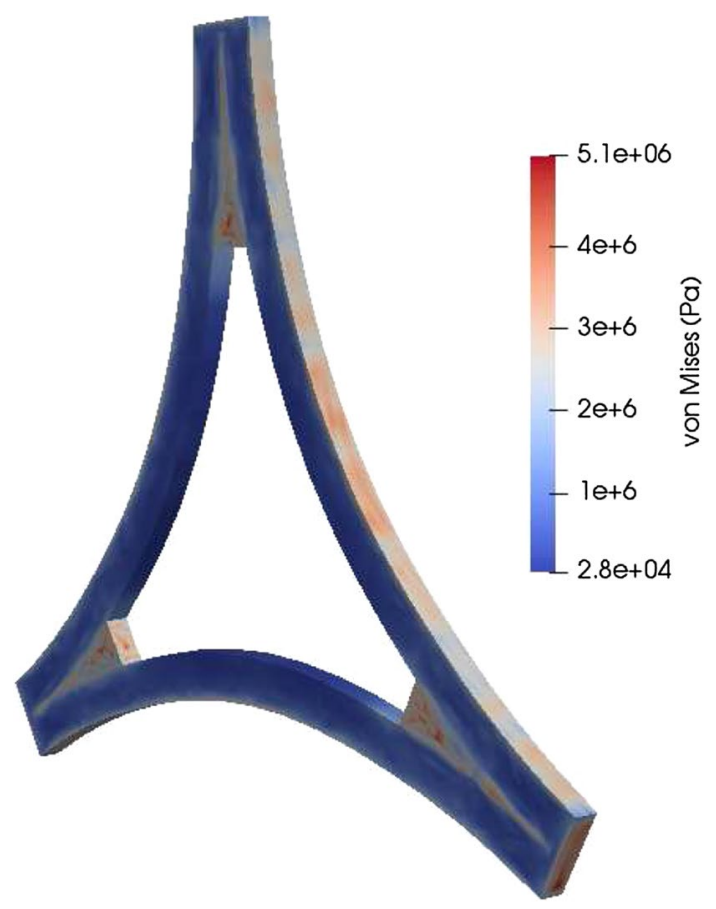

Fig. 5 von Mises stress on the deformed circular cell walls. Red patches indicate highest magnitude of von Misses stress, as indicated in the color bar

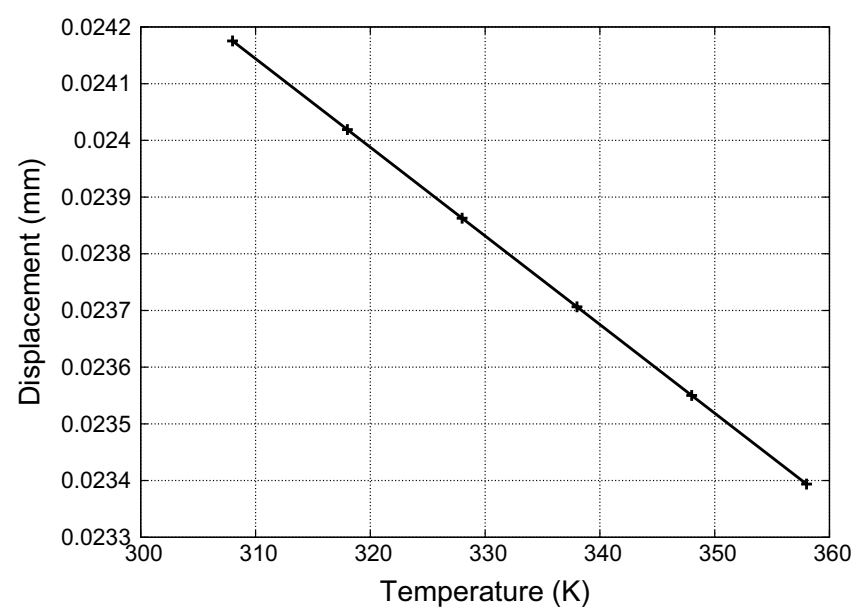

(a)
Fig. 6 , the maximum value of von Mises stress, both at the rims and at the meniscus, increases with increasing temperature of the hot junctions. Their values are found to be higher than the yield stress of beeswax which is $\approx 2 \times 10^{6}$ $\mathrm{Pa}[12,17,18]$. This clearly indicates that deformations of the cell walls are permanent. As a result of such permanent deformation in the vicinity of all the six triple junctions around a circular cell, the cell takes a rounded hexagonal shape, as shown schematically in Fig. 7.

In order to investigate how the above results get affected by slight change in the values of different thermomechanical properties for beeswax, we carry out the simulations with varying values of parameters, namely the coefficient of thermal expansion $\beta$, thermal conductivity $\kappa$, heat capacity $c_{p}$, density $\rho$, Poisson's ratio $v$, and Young's modulus $Y$. As we have now shown in Fig. 8, we vary the parameters around the experimental values, namely $\beta=344 \times 10^{-6} \mathrm{~K}^{-1}, \kappa=0.4 \mathrm{~W} \mathrm{~m}^{-1} \mathrm{~K}^{-1}, c_{\mathrm{p}}=2025 \mathrm{~J} \mathrm{~kg}^{-1}$, $\rho=970 \mathrm{~kg} \mathrm{~m}^{-3}, v=0.30$, and $Y=39 \times 10^{6} \mathrm{~Pa}$. We find that the displacement and von Mises stress do not vary with the variation of $\kappa, c_{\mathrm{p}}$ and $\rho$ while they depend on $\beta, v$, and $Y$. For all such variations (Fig. 8), the displacements are finite and the von Mises stress are greater than yield stress $(\approx 2 \mathrm{MPa})$, indicating a permanent deformation.

\section{Discussion and conclusion}

We carried out a set of computer simulations on the triple junction of three adjacent freshly built circular honeycomb cells. By taking beeswax as a constructing material, we demonstrated that at finite temperature difference in the vicinity of triple junctions, stress is generated in the adjacent circular walls due to which they straighten up.

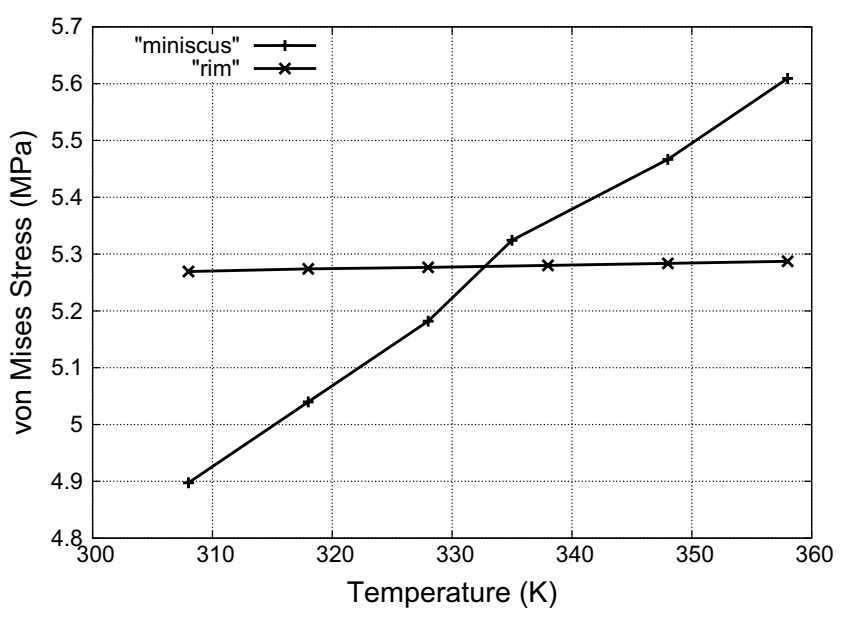

(b)

Fig. 6 Variation of a magnitude of displacement and $\mathbf{b}$ von Mises stress on the circular rims and meniscus with varying temperatures 


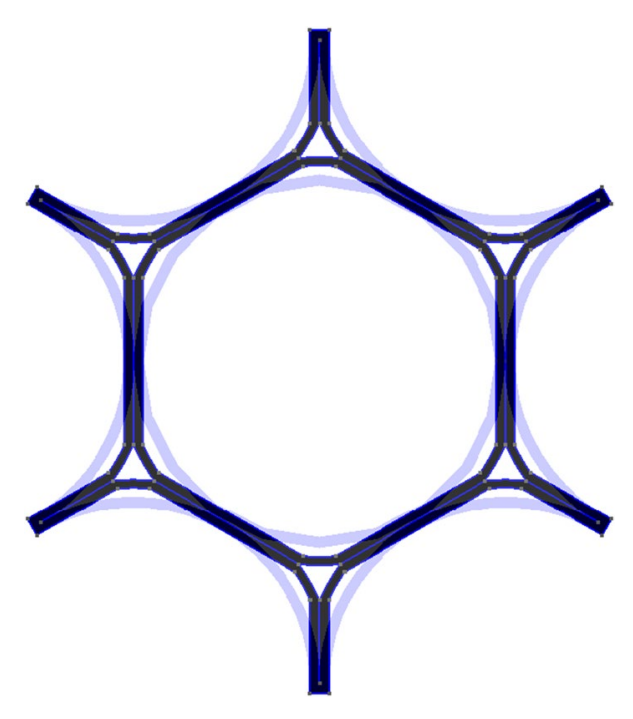

Fig. 7 A schematic drawing showing the deformation of initial circular cell configuration (shown shaded) due to the temperature gradient in the vicinity of all the six triple junctions

The cell walls are deformed permanently as quantified by the calculated von Misses stress. As a result of such deformation in the vicinity of all six triple junctions of a fresh circular cell, the rounded walls become elongated and the cell transforms to a rounded hexagonal shape depending on the temperature of the hot junctions. Thus, it is the temperature difference maintained by the bees within a comb and the thermomechanical properties of building material that lead to the hexagonal shape of the honeycomb cells.

The simplicity of our model lies on the fact that we have considered simply the conduction of heat and elastic deformation of beeswax under steady-state condition governed by heat conduction equation [Eq. (1)] and linear elastic model [Eq. (2)], respectively. In real scenario, it is known that bees deposit hot wax flakes on the junctions of two adjacent cell walls. However, the extent to which honeybees regulate their nest temperature during the building process is somewhat contradictory in the literature. Some experimental observations $[6,9,10]$ suggest that the temperature is relatively high (from 303 to $318 \mathrm{~K}$ ) in areas where the building activity takes place in comparison with the surrounding non-construction areas. In contrast, recent infrared and thermographic video observations of comb building [11] contradict the above observations and obtained the thorax temperature of working bees in between 305.45 and $312.75 \mathrm{~K}$ that generated wax temperature between 306.75 and $310.95 \mathrm{~K}$ during the construction of hexagonal cells. With such contradiction in experimental results, it is difficult to firmly establish any theoretical viewpoint. However, it is obvious from the present simulation that without reaching the melting point of beeswax, a finite deformation of circular walls can happen due to heat generated by the bees in the construction area.

In real scenario, as soon as the honeybees start depositing hot wax at the contact point of the circular rims, a temperature gradient is created. Eventually, the rims expand, the wax drop gets squeezed, and the hot wax starts flowing toward the center of the triple junction. Due to the flow of hot wax, the rims gets sealed with wax. As such, the contact point of the circular rims advances toward the center of the triple junction. The bees then start depositing hot wax at the new contact point and the whole process is repeated until the contact point gets close to the center of the triple junction. From this viewpoint, one can argue that honeybees transform the circular cells to rounded hexagonal cells by progressively bringing the circular rims closer starting from the contact point of the rims toward the center of the triple junction.

The above conclusion can be indirectly inferred from our simulation results in the following way: While measuring the von Mises stress, we found high values of von Mises stress at the outer surface of the rims and at the meniscus, as displayed in Fig. 5. The substantial amount of von Mises stress at the meniscus (4.9 MPa) indicates that the hot wax gets squeezed and would have flowed if the simulation could capture it. As our current computational experiment is designed only for a steady-state system, it could not capture the flow. In order to capture the flow, one needs to perform dynamic simulations that would be a computationally difficult task as compared to the present steady-state case. In order to simulate the spatiotemporal behavior of the transformation, the current finite element formulation needs to be changed to space-time finite element formulation $[19,20]$. Thus, the future prospects of the present formulation remain in simulating the dynamics in the vicinity of the triple junction through which one might gain better understanding of the transformation process. Although our present simulations yield a very small magnitude for maximum displacement (Fig. 4), it is enough to cause the said transformation if the flow of the molten wax is considered and the gap filling and sealing are assumed to be progressive.

Although our findings are in qualitative agreement with that of Karihaloo et al. [7] where a similar transformation was shown via numerical simulation of Maxwell model for viscoelastic beeswax, there is a noted difference between our model and that of Ref. [7]. The work of Karihaloo et al. is based on liquid equilibrium hypothesis [6], requiring molten wax to flow near the triple junction. In contrast, our simulation results suggested that it is not necessary to reach the melting point inside a comb cell to form a rounded hexagonal cell. It is the thermal expansion that takes place due to temperature gradient in the vicinity of 


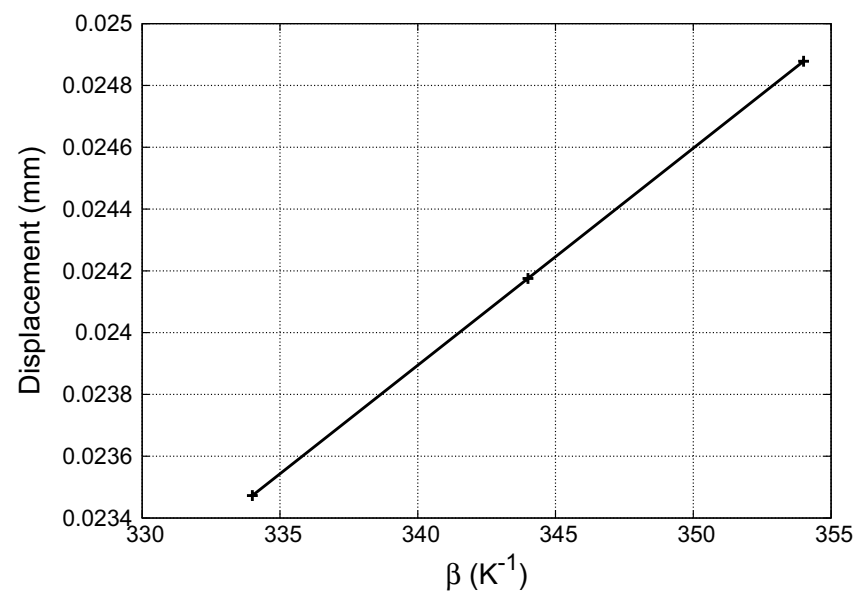

(a)

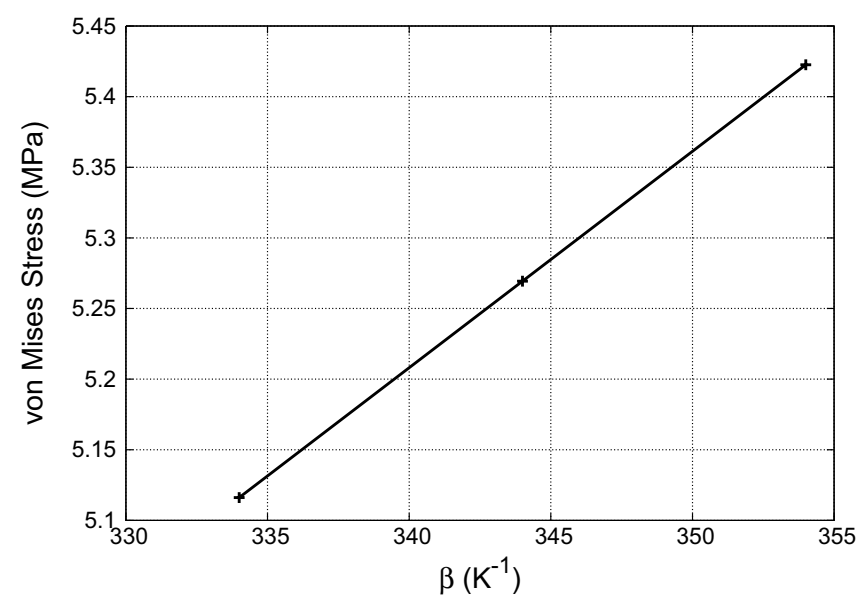

(c)

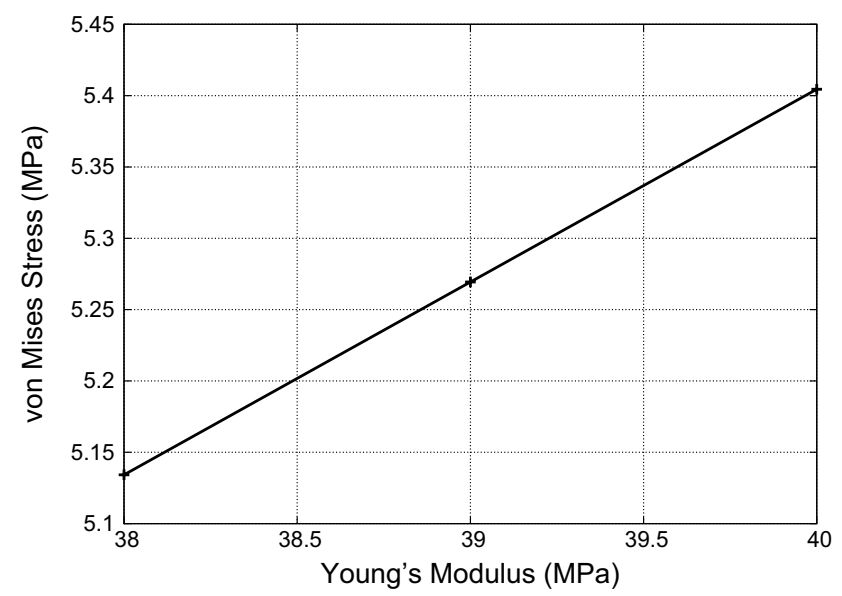

(e)

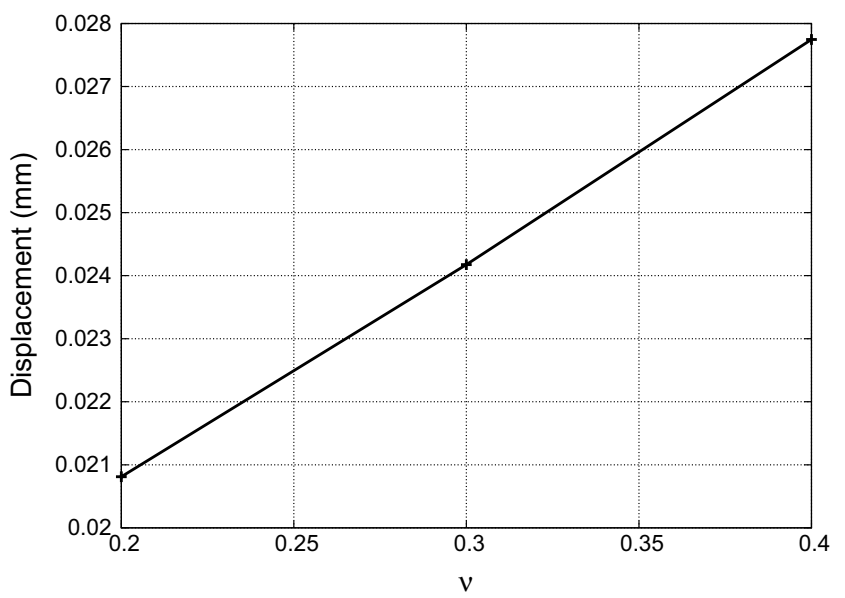

(b)

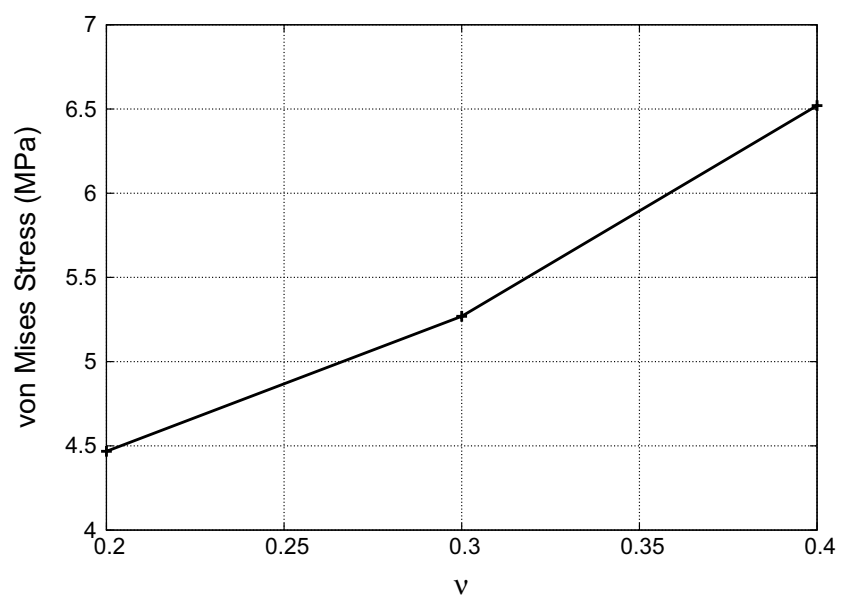

(d)

Fig. 8 Variation of magnitude of displacement and von Mises stress with the model parameters

a triple junction for which stress is generated and rounded walls gradually become elongated and hexagonal (Fig. 7).

As in Refs. [6, 7], the present simulation suggests that the hexagonal shape of the comb cells emerges solely due to the physical forces, and as such, bees do not have to perform any mathematical calculations or complex measurements of length and angles. However, in order to construct hexagonal cells, a perfect arrangement of 
isodiametric fresh circular cells is required in a way that each one is surrounded by six other similar cells [8]. A recent study [21] focuses attention on the initial structure of the honeycomb wherein, in order to understand how the honeybees construct the initial pattern, an agent-based model was formulated. By conducting twodimensional simulations, it was concluded [21] that the first stage of honeycomb construction can be understood as a self-organization process and the bees do not require to have any prior knowledge of the complex shape that they build. Thus, the mystery of honeybees' architecture lies in building the fresh isodiametric circular cells rather than the formation of hexagonal shape. Systematic observations showed that building errors occur if the cells are not surrounded by six other cells [8]. In that case, their final shape is not hexagonal but rather matches that of a polygon with as many sides as the number of surrounding cells. This indirectly confirms the influence of the preexisting pattern on the final shape of cells.

We would like to conclude by noting that although we are considering in this model pure beeswax as building material, there are other tribes of bee family, which construct hexagonal comb from mixture of pure wax and foreign substances [14]. By proper characterization of such building material, it would not be difficult to examine the structural transformation of comb cells with our present model formulation and we leave it as a future work.

\section{Compliance with ethical standards}

Conflict of interest The authors declare that they have no competing interests.

\section{References}

1. Tóth $F$ (1964) What the bees know and what they do not know. Bull Am Math Soc 70:468-481

2. Hales TC (2000) Cannonballs and honeycombs. Not AMS 47:440

3. Klarreich EG (2000) Foams and honeycombs. Am Sci 88:152
4. Hales TC (2001) The honeycomb conjecture. Discrete Comput Geom 25:1-22

5. Szpiro GG (2003) Kepler's conjecture. Wiley, Hoboken

6. Pirk CWW, Hepburn HR, Radloff SE, Tautz J (2004) Honeybee combs: construction through a liquid equilibrium process? Naturwissenschaften 91:350-353

7. Karihaloo BL, Zhang K, Wang J (2013) Honeybee combs: how the circular cells transform into rounded hexagons. J R Soc Interface 10:20130299

8. Nazzi F (2016) The hexagonal shape of the honeycomb cells depends on the construction behavior of bees. Sci Rep 6:28341

9. Hepburn HR, Kurstjens SP (1988) The combs of honeybees as composite materials. Apidologie 19:25

10. Bujok B, Kleinhenz M, Fuchs S, Tautz J (2002) Hot spots in the bee hive. Naturwissenschaften 89:299

11. Bauer D, Bienefeld K (2013) Hexagonal comb cells of honeybees are not produced via a liquid equilibrium process. Naturwissenschaften 100:45

12. Zhang K, Duan HL, Karihaloo BL, Wang J (2010) Hierarchical, multilayered cell walls reinforced by recycled silk cocoons enhance the structural integrity of honeybee combs. Proc Natl Acad Sci USA 107:9502

13. Hepburn RH (1986) Honeybees and wax. Springer, Berlin

14. Buchwald R, Breed MD, Greenberg AR (2008) The thermal properties of beeswaxes: unexpected findings. J Exp Biol 211:121

15. Craig RG, Eick JD, Peyton FA (1965) Properties of natural waxes used in dentistry. J Dent Res 44:1308

16. Hill R (2004) The mathematical theory of plasticity. Clarendon Press, Oxford

17. Buchwald R, Breed MD, Greenberg AR, Otis G (2006) Interspecific variation in beeswax as a biological construction material. J Exp Biol 209:3984

18. Buchwald R, Breed MD, Bjostad L, Hibbard BE, Greenberg AR (2009) The role of fatty acids in the mechanical properties of beeswax. Apidologie 40:585

19. Huang $\mathrm{H}$, Costanzo $\mathrm{F}$ (2002) On the use of space-time finite elements in the solution of elasto-dynamic problems with strain discontinuities. Comput Methods Appl Mech Eng 191:5315

20. Dumont S, Jourdan F, Madani T (2018) 4D remeshing using a space-time finite element method for elastodynamics problems. Math Comput Appl 23:29

21. Narumi T, Uemichi K, Honda H, Osaki K (2018) Self-organization at the first stage of honeycomb construction: analysis of an attachment-excavation model. PLoS ONE 13(10):e0205353

Publisher's Note Springer Nature remains neutral with regard to jurisdictional claims in published maps and institutional affiliations. 